\title{
Revisiting Physical Examination: Abadie's Sign and Achilles Intratendinous Changes in Subjects with Diabetes
}

\author{
Michele Abate $^{a} \quad$ Cosima Schiavone $^{a} \quad$ Vincenzo Salini $^{a} \quad$ Isabel Andiab \\ a Department of Medicine and Science of Aging, University G. d'Annunzio, Chieti-Pescara, Chieti, Italy; \\ ${ }^{b}$ BioCruces Health Research Institute, Cruces University Hospital, Barakaldo, Spain
}

\section{Key Words}

Abadie's sign · Achilles tendon · Diabetes mellitus .

Ultrasonography

\begin{abstract}
Objective: The aim of this study was to evaluate whether or not the positivity of Abadie's sign could be an indicator of asymptomatic Achilles intratendinous changes. Subjects and Methods: A total of 18 patients (36 tendons) suffering from diabetes, with at least 1 Achilles tendon positive to Abadie's sign, were compared to matched subjects with diabetes bilaterally negative to Abadie's sign. Anthropometric measures and the Toronto Clinical Neuropathy Score were registered. Echotexture was evaluated and degenerative features classified as absent, mild, moderate and severe. The frequencies of structural abnormalities, according to both Abadie's sign and the Toronto Clinical Neuropathy Score, were determined. Results: In the first group 26 out of 36 tendons (72.2\%) showed positive Abadie's sign and a significantly higher frequency of moderate and severe (65.3\%) structural abnormalities compared to Achilles tendons with negative sign $(4.3 \% ; p<0.0001)$. This frequency was similar to that observed in the subjects with the highest Toronto Clinical Neuropathy Score (64.2\%). Conclusions: This study showed that Abadie's sign was a useful tool for assisting in the diagnosis of asymptomatic Achilles intratendinous changes, which, when detected early, could help prevent
\end{abstract}

unexpected tendon rupture. The concordance between Abadie's sign and Achilles sonographic abnormalities needs to be evaluated in a larger sample to consider it useful for practical purposes.

(c) 2013 S. Karger AG, Basel

\section{Introduction}

The world prevalence of diabetes is $6.4 \%$, affecting 285 million adults in 2010, and this number is anticipated to increase by $69 \%$ in developing countries and by $20 \%$ in developed countries by 2030 [1]. Diabetes is frequently associated with several rheumatological disorders such as shoulder adhesive capsulitis, trigger finger, osteoarthritis, carpal tunnel and crystalline arthropathy [2], as well as tendon degeneration [3], as reported in studies on plantar fascia and Achilles tendon [3-5].

In the past century, Joseph Abadie observed that 'pinching of, or the application of firm pressure to the Achilles tendon does not result in pain in tabes dorsalis. This is because the sense of deep pain has been abolished' [6]. Afterwards, the Abadie's sign was found positive in every neurological condition characterized by deep sensitivity loss. Hence, the aim of this study was to evaluate whether or not the positivity of Abadie's sign could be an indicator of asymptomatic Achilles intratendinous changes in subjects with diabetes. 


\section{Subjects and Methods}

The Institutional Review Board approved this study, and informed consent was obtained from each subject. The participants included in the study were patients with diabetes seeking care for musculoskeletal upper limb disorders who were referred to our Ultrasound Service. The subjects enrolled were considered to have some predisposition to tendon disorders.

Demographic, anthropometric and clinical data were recorded. The Abadie's sign was incited bilaterally by vigorously pinching the Achilles tendon at the midportion between the thumb and the forefinger [6]. The sign was considered positive when the subject felt only a sensation of pressure without any evident discomfort (VAS $\leq 3$ ), and negative when he/she declared evident pain (VAS $\geq 4$ ). For every subject positive to Abadie's sign, a subject with diabetes, matched for sex and age but bilaterally negative to the sign, was recruited.

Sensory-motor neuropathy was assessed using the Toronto Clinical Neuropathy Score (TCNS) [7], which is the summed total of three scores (range: 0-19) as follows: (1) symptoms (foot pain, numbness, tingling, weakness, ataxia and upper limb symptoms graded as $1=$ present, $0=$ absent $),(2)$ knee and ankle reflexes $(2=$ absent, 1 = reduced, $0=$ normal) and (3) sensory test score (pinprick, temperature, light touch, vibration and position sense; $1=$ abnormal, $0=$ normal).

The ultrasound evaluation of Achilles tendon was performed (M.A.) using a high-resolution, multifrequency linear array transducer.

On the basis of structural abnormalities [4], tendons were stratified for severity as 'mild' (one area of disorganized echotexture), 'moderate' (some areas of disorganized echotexture) and 'severe' (disorganized echotexture + diffuse hypo- or hyperechoic areas and/or calcifications). The distribution of lesions according to severity along the spectrum of TCNS were calculated as upper (11$16)$, intermediate (6-10) and low (1-5) or according to Abadie's sign.

The $\chi^{2}$ test was used to evaluate associations between categorical data. Cohen's kappa coefficient was used to evaluate the concordance between the positivity of Abadie's sign and the severity of lesions at the ultrasound evaluation. Data are reported as mean $\pm \mathrm{SD}$ (as frequencies and percentages).

\section{Results}

The demographic, anthropometric and clinical data are reported in table 1 . Of the 18 subjects, $8(44.4 \%)$ had a bilateral positivity of the sign; in 10 patients the sign was negative on the contralateral side. Hence, of the 36 Achilles tendons of the 18 patients, $26(72.2 \%)$ were positive to Abadie's sign while 10 (27.8\%) tendons were not.

Major structural lesions were more prevalent in Achilles tendons positive to Abadie's sign than in those with negative sign (table 1). In the different classes of TCNS (upper, intermediate and lower), 14, 24 and 34 Achilles tendons were included, respectively; ultrasound features
Table 1. Clinical features and frequencies of structural abnormalities, according to both Abadie's sign and Toronto Clinical Neuropathy Score

\begin{tabular}{llll}
\hline & \multicolumn{2}{l}{ Abadie's sign } & \\
\cline { 2 - 4 } & positive & negative & $\mathrm{p}$ \\
\hline Subjects & 18 & 18 & - \\
M/F ratio & $10 / 8$ & $10 / 8$ & - \\
Age, years & $60.7 \pm 5.3$ & $59.9 \pm 5.9$ & n.s. \\
Duration of diabetes, years & $10.2 \pm 3.5$ & $6.9 \pm 3.4$ & 0.001 \\
BMI & $24.9 \pm 3.4$ & $25 \pm 2.6$ & n.s. \\
TCNS & $9.9 \pm 3.7$ & $4.3 \pm 2.2$ & 0.000 \\
Number of tendons & 26 & 46 & - \\
Absent degeneration & $5(19.2 \%)$ & $28(60.8 \%)$ & 0.0007 \\
Mild degeneration & $4(15.3 \%)$ & $16(34.7 \%)$ & n.s. \\
Moderate degeneration & $11(42.3 \%)$ & $2(4.3 \%)$ & 0.0001 \\
Severe degeneration & $6(23 \%)$ & - & 0.0007 \\
\hline & TCNS Class & & \\
\cline { 2 - 5 } & upper & intermediate & lower \\
& $(11-16)$ & $(6-10)$ & $(1-5)$ \\
\hline Severe degeneration & 14 & 24 & 34 \\
Mbsent degeneration & 0 & $11(45.8 \%)$ & $22(64.7 \%)$ \\
Mild degeneration & $5(35.7 \%)$ & $8(33.3 \%)$ & $7(20.5 \%)$ \\
& $6(42.8 \%)$ & $3(12.5 \%)$ & $4(11.7 \%)$ \\
& $3(21.4 \%)$ & $2(8.3 \%)$ & $1(2.9 \%)$ \\
\hline
\end{tabular}

of moderate and severe degeneration prevailed in tendons belonging to the highest class of TCNS (table 1).

A good concordance (Cohen's kappa coefficient = 0.65 ) between the positivity of Abadie's sign and severe and moderate ultrasound lesions was found.

\section{Discussion}

The high percentage (72.2\%) of Abadie's sign in this study could be considered an indicator of asymptomatic Achilles intratendinous changes in subjects with diabetes because high frequency of moderate-to-severe sonographic tendon abnormalities was seen in these patients. Several factors could explain this finding. Patients with diabetes and sensory-motor neuropathy are likely to have unperceived traumas and to have altered gait biomechanics; this complication is usually associated with a longer duration of the disease, which is a concurrent pathogenetic mechanism [8].

Tendon degeneration results from the formation of advanced glycation end products and from the subsequent cross-linking within collagen fibers, which can cause deterioration of the biological and mechanical 
function [3, 4]. An increased generation of reactive oxygen species and a sustained upregulation of proinflammatory and proapoptotic mediators are also present $[3-5,9]$.

This study showed that Abadie's sign had a very good correlation with the highest TCNS. A probable explanation could be the intrinsic pathogenetic mechanisms of the test, which relies on the interruption of the pathways carrying the deep pain sensitivity to the perception areas in the brain [10]. Indeed, TCNS evaluates several clinical sensory-motor components and, due to the large clinical variability of diabetic neuropathy, subjects with prevalent loss of surface sensitivity, absence of reflexes and less/absent impairment of deep sensitivity fibers can have high TCNS.

Most importantly, Abadie's sign offers a relevant clinical advantage because it may be easily and rapidly performed in comparison to TCNS, which is time consuming. The limitations of this study include, firstly, the highly subjective nature of Abadie's sign, which depends on the individual's threshold of pain perception. Secondly, it may be due to several neurological lesions which could be present in patients with long duration of diabetes. Thirdly, the loss of sensitivity of Achilles tendon may be executed, at least partly, secondary to its intrinsic degenerative pathology, whatever its pathogenesis. Indeed, it is possible that, when the tendon undergoes degenerative changes, its neural component may be affected and therefore its sensitivity may be reduced. If this were the case, the positive Abadie's sign could be secondary to a common tendinopathy without any relationship with diabetic neuropathy.
This study has clinical relevance because it shows that a simple and quick maneuver can allow identification of a high percentage of subjects with diabetes affected by silent Achilles intratendinous changes. This statement is limited to subjects with diabetes and peripheral neuropathy, which is a frequent complication, but cannot be extended to all patients with diabetes. Early detection is important because it could help prevent unexpected tendon rupture, which may happen in the absence of pain. This would be particularly true in diabetic patients consuming other drugs that have detrimental effects on the Achilles tendon, such as fluoroquinolones [11]. However, our data must be considered as mere empirical observations, which need confirmation in larger samples. In particular, further studies are needed to assess the prevalence of Abadie's sign in subjects without diabetes but with sonographic features of Achilles tendon damage.

\section{Conclusion}

This study showed that Abadie's sign was a useful tool for assisting in the diagnosis of asymptomatic Achilles intratendinous changes, which, when detected early, could help prevent unexpected tendon rupture. The concordance between Abadie's sign and Achilles sonographic abnormalities needs to be evaluated in a larger sample to consider it useful for practical purposes.

\section{Disclosure Statement}

The authors declare no conflict of interest.

\section{References}

1 Shaw JE, Sicree RA, Zimmet PZ: Global estimates of the prevalence of diabetes for 2010 and 2030. Diabetes Res Clin Pract 2010;87: 4-14.

2 Burner TW, Rosenthal AK: Diabetes and rheumatic diseases. Curr Opin Rheumatol 2009;21:50-54.

-3 Abate M, Schiavone C, Pelotti P, et al: Limited joint mobility in diabetes and ageing: recent advances in pathogenesis and therapy. Int J Immunopathol Pharmacol 2010;4:997.

4 Abate M, Schiavone C, Di Carlo L, et al: Achilles tendon and plantar fascia in recently diagnosed type II diabetes: role of body mass index. Clin Rheumatol 2012;31:1109-1113.
5 Batista F, Nery C, Pinzur M, et al: Achilles tendinopathy in diabetes mellitus. Foot Ankle Int 2008;29:498-501.

6 Joseph Louis Irenée Jean Abadie. Gazette hebdomadaire des sciences médicales de Bordeaux 1905;26:408.

7 Bril V, Perkins BA: Validation of the Toronto Clinical Scoring System for diabetic polyneuropathy. Diabetes Care 2002;25:2048-2052.

8 Giacomozzi C, D’Ambrogi E, Uccioli L, et al: Does the thickening of Achilles tendon and plantar fascia contribute to the alteration of diabetic foot loading? Clin Biomech (Bristol, Avon) 2005;20:532-539.
9 Alikhani Z, Alikhani M, Boyd CM, et al: Advanced glycation end products enhance expression of pro-apoptotic genes and stimulate fibroblast apoptosis through cytoplasmic and mitochondrial pathways. J Biol Chem 2005; 280:12087-12095.

10 Tamer A, Yildiz S, Yildiz N, et al: The prevalence of neuropathy and relationship with risk factors in diabetic patients: a single-center experience. Med Princ Pract 2006;15:190 194.

11 Yu C, Giuffre B: Achilles tendinopathy after treatment with fluoroquinolone. Australas Radiol 2005;49:407-410. 